\title{
First VLBI observations of methanol maser polarisation, in G339.88-1.26
}

\author{
R. Dodson \\ Observatorio Astronómico Nacional, Apartado 112, Alcala de Henares 28803, Spain \\ e-mail: r.dodson@oan.es \\ Received 14 September 2007 / Accepted 21 December 2007

\section{ABSTRACT}

\begin{abstract}
Aims. We investigate class II methanol masers and the environment in which they form with the Long Baseline Array (LBA). Methods. Using full polarisation VLBI, we were able to measure the magnetic field directions so as to distinguish between the two main models of the environment in which methanol masers form: disks or shocks.

Results. We present polarised images of the methanol maser source G339.88-1.26, made with the LBA at 6.7-GHz. With these first polarisation maps made with the LBA, which successfully reproduce observations with the ATCA confirming the new AIPS code, a new technique for Southern VLBI is opened. The magnetic field directions found are inconstant with methanol masers arising in disks for the majority of the emission.
\end{abstract}

Key words. techniques: polarimetric - masers - radio lines: stars - stars: formation - stars: individual: G339.88-1.26

\section{Introduction}

Massive stars play a crucial role in the evolution of galaxies, but the processes leading to massive star formation are poorly understood, e.g. Garay \& Lizano (1999), McKee \& Tan (2003). One of the major questions is whether or not they form from the accretion or via congregation of previously formed smaller bodies. Masers, being compact, bright, and at exact frequencies, are sensitive to the local physical environment, making them ideal probes of the areas in and around massive star-forming regions. Interstellar masers of three molecules, $\mathrm{H}_{2} \mathrm{O}, \mathrm{OH}$ and methanol, are both powerful and widely associated with active high-mass star formation regions (Forster \& Caswell 1989; Menten 1991). However, both $\mathrm{H}_{2} \mathrm{O}$ and $\mathrm{OH}$ masers are quite common and also found towards other objects such as low mass star-forming regions (Furuya et al. 2001), evolved stars (Wilson \& Barrett 1972) and the centres of galaxies (Dos Santos \& Lepine 1979), unlike methanol masers which are exclusively linked to high mass star formation (Minier et al. 2003).

The two strongest class II methanol masers (i.e. those closely associated with $\mathrm{OH}$ masers and strong far-infrared emission) were discovered at $12.179 \mathrm{GHz}$ by Batrla et al. (1987) and at $6.669 \mathrm{GHz}$ by Menten (1991). These methanol masers are closely associated with high-mass star forming regions (Norris et al. 1988; Walsh et al. 1997). The brightness of methanol masers allows high resolution interferometric observations to be made, which provide accurate measurements of position, velocity and dimensions of the individual maser components. Previous interferometric observations have generally used connected element interferometers (Norris et al. 1993; Phillips et al. 1998; Walsh et al. 1998) that offer resolutions of the order of arcseconds (which can be improved upon by super-resolving the velocity features, see Phillips et al. 1998), though a number of VLBI (Very Long Baseline Interferometry) experiments (e.g. Menten et al. 1988, 1992; Norris et al. 1998; Minier et al. 2000; Moscadelli et al. 1999; Dodson et al. 2004), which provide milliarcsecond resolution, have been performed. The VLBA covers 12.2-GHz, while the Australian Long Baseline Array (LBA) and the European VLBI Network (EVN) cover the 6.7-GHz transition. Furthermore high resolution, connectedelement interferometry, imaging has been done with MERLIN (Vlemmings et al. 2006) (6.7-GHz) and the Parkes-Tidbinbilla Interferometer (Norris et al. 1988) (12.2-GHz). One of the early successes of methanol maser imaging was to provide strong support for the accretion model. It was claimed from such observations that methanol masers formed predominately linear structures, and these marked the accretion disks.

Many of the observed masing sites have individual masers located along lines or arcs, often with a near-monotonic velocity gradient along the line. It was suggested (Norris et al. 1993, 1998; Phillips et al. 1998) that these linear structures trace masers embedded in an edge-on disk surrounding a young star. Values of disk radii and enclosed masses, derived from modelling these sources assuming Keplerian rotation, agree with theoretical models of accretion disks around massive stars (Lin \& Pringle 1990; Hollenbach et al. 1994). Recently a subsection of one methanol maser has been extensively and successfully modelled as a disk (Pestalozzi et al. 2004) amplifying a background source, but on the whole these models do not satisfactorily explain the majority of methanol masers.

Whilst Norris et al. claimed the observations demonstrated they delineated disks, Walsh et al. (1998) found only 36 of 97 maser sites to be linearly extended. While the circumstellar disk hypothesis is consistent with their data, they considered it unlikely as the derived values of the enclosed masses are too high in some cases. They suggested the linear geometry of $6.7-\mathrm{GHz}$ methanol masers resulted from shocks with a small, smooth, velocity gradient, as a consequence of the distance of the masers from the shock origin. Norris et al. (1998) had rejected this as a possible explanation as they believed shocks could not produce the velocity gradient they saw in many of their maser sites. 
Edge-on shock and edge-on disk models produce linear features across the sky but shock fronts are not normally so well ordered. At mas resolutions we probe structures of the order of tens of $\mathrm{AU}$, the perfect scale to investigate these differences. One further crucial difference is that the first model would have the magnetic field across the major axis of the feature - as the disk is formed by matter collapsing along the field lines (Mouschovias \& Ciolek 1999), and the second will have it along the axis, due to entrainment of the field at the shock front. Therefore measuring the field directions could distinguish between these two models.

Detailed studies (Watson 1994; Elitzur 1996; Gray 2003) of the polarisation properties of $\mathrm{OH}, \mathrm{H}_{2} \mathrm{O}$ and $\mathrm{SiO}$ masers have been made, but there has been relatively little investigation of the polarisation properties of methanol masers. Single dish observations of polarisation in methanol masers are all from the strongest sources: Koo et al. (1988) observed W3(OH) and NGC 6334F at $12 \mathrm{GHz}$, while Caswell et al. (1995) determined that the level of circular polarisation for a number of strong $6.7 \mathrm{GHz}$ methanol maser features was less than $1 \%$. Ellingsen (2002) reported on $6.7 \mathrm{GHz}$ polarisation observations with the ATCA of NGC 6334F, where he found linear polarisation fractions of up to $10 \%$. The first high resolution polarisation images were made with MERLIN at a resolution of 50 mas (Vlemmings et al. 2006), of $\mathrm{W} 3(\mathrm{OH})$, where polarisation fractions of upto $8 \%$ but typically $2-3 \%$ were found. Those polarisation angles were consistent with those of the OH-masers, and furthermore lie along the methanol maser emission as expected for shock excited regions.

\subsection{G339.88-1.26}

The maser G339.88-1.26 was discovered at $12 \mathrm{GHz}$ by Norris et al. (1987), and at $6.7 \mathrm{GHz}$ by MacLeod et al. (1992). Follow-up at arcsecond resolution by the Australia Telescope Compact Array (ATCA) showed strong emission with a roughly linear morphology and a monotonic velocity gradient (Norris et al. 1993). Ellingsen et al. (1996) detected weak radio continuum emission which peaks at the same position as the maser emission. The Mid Infra-Red (MIR) observations at $10 \mu \mathrm{m}$ of Stecklum (1998) detected elongated emission along the same position angle as the disk inferred by the methanol masers. These combined to make this one of the best candidates for the disk model for methanol masers, with the masers formed in the dusty disk hiding an embedded massive, forming, star. However higher resolution, 10 and $18 \mu \mathrm{m}$ MIR observations, at the Keck Observatory (De Buizer et al. 2002) resolved the IR source into three components. VLBI observations from 1996, included in the same paper, showed that the masers formed an inverted $Y$ shape and lay between $1 B$ and 1C. See Fig. 2b in that paper. That is they do not mark the disk, indeed there was no disk, nor do they have a Keplerian velocity distribution.

\section{Development of new Mount types in AIPS}

As a telescope tracks a source across the sky the angle of the source on the sky to that of the telescope feed changes. When observing with Left Circular and Right Circular Polarisations (LCP and RCP) this has the effect of introducing a phase shift between the two recorded data-streams. To post-process these data these phases need to be removed, and the feed impurities need to be solved for and included in the calibration. A good summary of the steps needed to make polarisation VLBI images can be found in Aaron (1997). In this study, and indeed all other sources on VLBI polarisation, the feed mounts are assumed to be Cassegrain or Equatorial. Different mounts rotate the feeds in different fashions on the sky as the telescope tracks a source. Two new mount types to those supported have been added.

For VLBI observations AIPS (Greisen 1988) remains the only tool for data calibration, therefore this has been our target for the extension of mount types. The code to support the Nasmyth mount type and the E-W mount type have been developed. The latter is a subset of the X-Y mount (traditionally for Low Earth Orbit satellite tracking stations) where the second axis lies East-West. The only example known to the author is the Hobart telescope which is part of the Australian Long Baseline Array (LBA). The alternative configuration is the N-S mount, where the second axis lies North-South. The "keyhole", where large angular changes are required for small movements on the sky, falls on the second axis. Compared the Alt-Az mount, the $\mathrm{X}-\mathrm{Y}$ mounts move the keyhole from the Zenith to the horizon, where observations are not normally made. The second mount type added, which is not used in the data presented here, is for the Nasmyth type. The Nasmyth mount is more normally used on optical instruments, as it allows space for very large instrumentation packages. Until now this has not been needed for Radio Telescopes, but new quasi-optical systems allow the siting of multiple feeds for different bands at the Nasmyth foci. This configuration allows the co-observing of widely separated observing bands, which is particularly useful for the calibration of mm-VLBI (Dodson \& Rioja 2008). The new code is for the new mm-VLBI telescope being constructed at Yebes, Spain, which will cover frequencies from $2 \mathrm{GHz}$ to $115 \mathrm{GHz}$, and also the IRAM telescope at Pico Veleta (Spain). Left handed and Right handed Nasmyth foci are also included in the new AIPS code, as required for the Left or Right optical branch. Full details can be found in Dodson (2007). These observations with the LBA were used to test EW-mount portion of these new subroutines.

When the LBA results on G339.88-1.26 are compared to those of ATCA observations (Ellingsen, Priv. Comms.), made in Sept. 1999, an excellent match in the linear polarised fluxes and angles is found, after including the absolute polarisation angle offset between the brightest polarised component (at $-38.7 \mathrm{~km} \mathrm{~s}^{-1}$ ). See Fig. 1 where the position angles, linear polarised and total flux are compared for the two instruments. ATCA values are plotted with red closed circles, and the LBA values with blue open boxes. The values are extracted from a sum across the $Q$ and $U$ images (with miriad's imspec) which allows the comparison of these two datasets with very different resolutions. The errors are absolute errors from the confidence in the polarisation calibration, the relative errors are much less. Note that Goedhart et al. (2004) report this source as "not significantly variable", with one component falling $\left(-32.39 \mathrm{~km} \mathrm{~s}^{-1}\right)$ and one rising $\left(-33.19 \mathrm{~km} \mathrm{~s}^{-1}\right)$. Both of these appear in the Western cluster, which is where we find great emission compared to 1999. There is also a short-fall in flux (total and linear) from between -38.5 and $-38 \mathrm{~km} \mathrm{~s}^{-1}$.

\section{Observations}

Observations for this experiment, V148, were performed in two sessions, V148A in 25 April and V148B in 23 July 2001. Observations were made of the calibrators; 1718-649 and 1610-771 plus 1921-293 and 1334-127 for fringe and absolute position angle calibration and three target masers; NGC 6334F, G327.120+0.511, G339.88-1.26. Unfortunately in the first set of observations Hobart failed, leaving only four antennae; Parkes, Mopra, ATCA, and Ceduna. These were not sufficient to provide good polarisation calibration. Only total power observations 


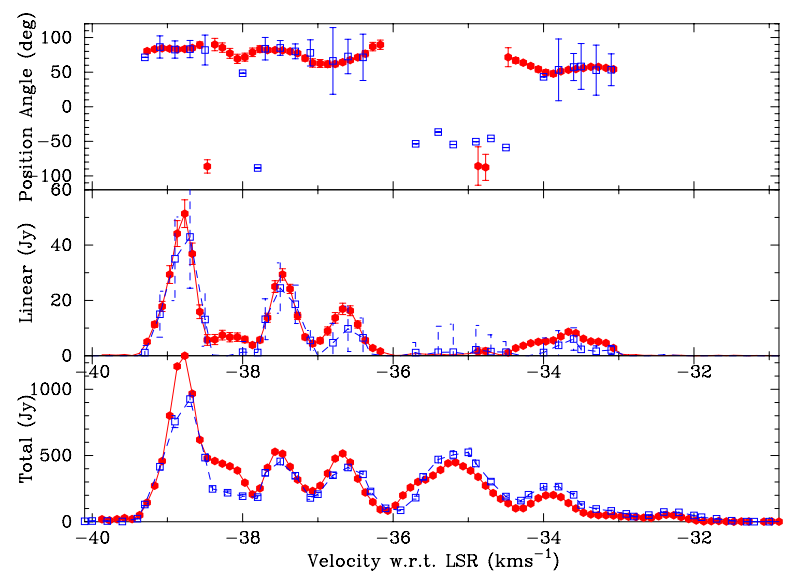

Fig. 1. Polarisation angle, linear and total flux for G339-1.26, as observed by the LBA (this paper, blue open squares) and the ATCA (Ellingsen, priv. comm., red closed circles). The spectra is scalar summed across the image (Stokes $I, Q$ and $U$ ), and shows good agreement between the VLBI and the connected array results. The found Stokes $I$ flux from the 2001 observations is slightly higher between $\left(-34.5\right.$ to $\left.-33.0 \mathrm{~km} \mathrm{~s}^{-1}\right)$, whereas the fractional polarisation is slightly lower. This is the region found to be varible in Goedhart et al. (2004). The errors are the absolute errors based on the confidence in the polarisation calibration ( $2 \%$ and $0.4 \%$ respectively), not the relative errors. Where errors are not shown they could not be calculated.

could be extracted from this session. The V148B observations included Hobart and could be calibrated. Of these observations only G339.88-1.26 produced interesting results, so this paper only reports on that source. The other two targets were effected by a range of correlator issues, and will be reobserved. The data recorded were $4 \mathrm{MHz}$ of 2 bits, dual polarisation, and all four stokes and 1024 channels were formed at the LBA correlator. The data sampling was two seconds, and the autocorrelations were also recorded to aid calibration with the AIPS task ACFIT. A problem with the sampler levels, which leads to ringing across the bandpass, meant that the data had to be Hanning smoothed over adjacent channels, giving an effective velocity range of $0.35 \mathrm{~km} \mathrm{~s}^{-1}$. The resolution of these observations is $3.1 \times 2.8$ mas, with a position angle of $49^{\circ}$. The rms for a single channel, unaffected by residual flux, is $0.1 \mathrm{Jy}$.

The calibration was done via the normal routes as described in Diamond (1989), and as reported in Dodson et al. (2004). Delay and rate calibration was done in AIPS, and then final self-calibration and model-fitting in difmap (Shepherd 1997). In addition polarisation imaging was done in Miriad (Sault et al. 1995), on the difmap self-calibrated data. AIPS tasks are refered to in uppercase bold, and Miriad tasks in lowercase bold. 1718-649, being a point-like source, provided the final amplitude calibration as the flux (2.7 Jy) was known from ATCA observations. The polarisation corrections were made with the total intensity models of the sources 1718-649 and 1610-771 derived from both epochs, using the code with new mount types defined, and the task LPCAL. As this is the first demonstration of the code great care has been taken to ensure that the phase corrections produce the expected behaviour, on this experiment and also on others available (V182). See Dodson \& Rioja (2008) for details of the analysis. The polarisation corrections, the Dterms, were found to be very high - as high as $20 \%$ for some antennae - which could perhaps be expected on this very early full-polarisation VLBI experiment for the LBA. Recent results (from the e-VLBI real time monitoring) indicate that the polarisation purity has improved greatly. Nevertheless, the estimated

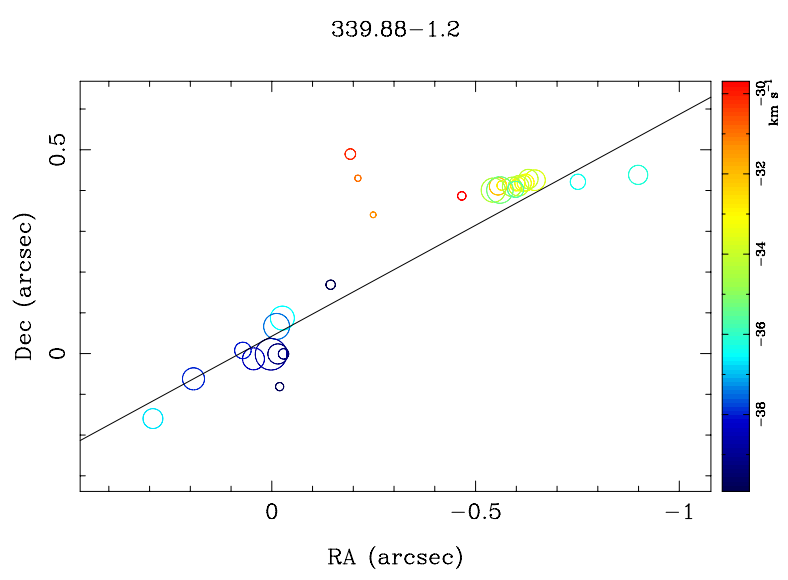

Fig. 2. Spot plot of G339.88-1.26. The sizes represent the square root of the component flux and the colour the velocity. There are three clusters of points, to the North, the East and the West. The position errors are much less than the symbol sizes. The line shows the best fit to the disk, at $-61^{\circ}$.

accuracy of our polarisation calibration is about $2 \%$, based on the rms difference between solutions on the two different calibrators - both assuming that the sources were polarised and unpolarised. The data cubes were formed at full spectral resolution (even though the data had been Hanning smoothed) at 0.5 mas/pixel in difmap. All channels were searched for emission across the source, and simple Gaussian models were fitted where emission was found. The data was self-calibrated on the strongest compact emission (at $-37.5 \mathrm{~km} \mathrm{~s}^{-1}$ ) to the model. The data were exported to Miriad, imaged at $0.7 \mathrm{mas} / \mathrm{pixel}$ and cleaned around the regions where the difmap models were found. The regions in which no emission had been found were blanked. Deconvolved images of Stokes $I, Q$ and $U$ were generated and polarisation images were derived from these, clipped at a position angle error of $5^{\circ}$, using impol. The polarisation images presented are from the images integrated over frequency, as the spots are isolated in space and velocity. This allowed more compact datasets to be formed without loss of information. There is little noticeable difference between the images from the entire datacube and the integrated one.

\section{Stokes I images of G339.88-1.26}

We present Stokes I images of the source in Figs. 2 and 3, prepared using msplot ${ }^{1}$. Figure 2 is a "spot plot" derived from the models fitted to the uv-data in Difmap. This allows us to present a noise free image, with all the components clearly identified. The velocities of the components are colour coded, whilst the square root of the flux/beam sets the circle size. This provides both a comparison to other, similar, papers (e.g. Dodson et al. 2004) and a guide to the arrangement of the different features, whilst not suppressing the weaker features. The map is self calibrated to the strong compact feature at $-37.5 \mathrm{~km} \mathrm{~s}^{-1}$, and this then falls at the origin. We find the lowest absolute velocity, and weakest, spot positions to the North. These run from -28 to $-31.5 \mathrm{~km} \mathrm{~s}^{-1}$, with most negitive velocities furthest from the centre. This is also the brightest feature peaking at $9 \mathrm{Jy} /$ beam. The Western cluster starts at $-32.5 \mathrm{~km} \mathrm{~s}^{-1}$, and runs further westwards with velocity until $-33.5 \mathrm{~km} \mathrm{~s}^{-1}$. After which the spots track back across the same positions in the sky. There is

\footnotetext{
1 http://www .atnf.csiro.au/people/Chris.Phillips/ software.html
} 
$339.88-1.2$

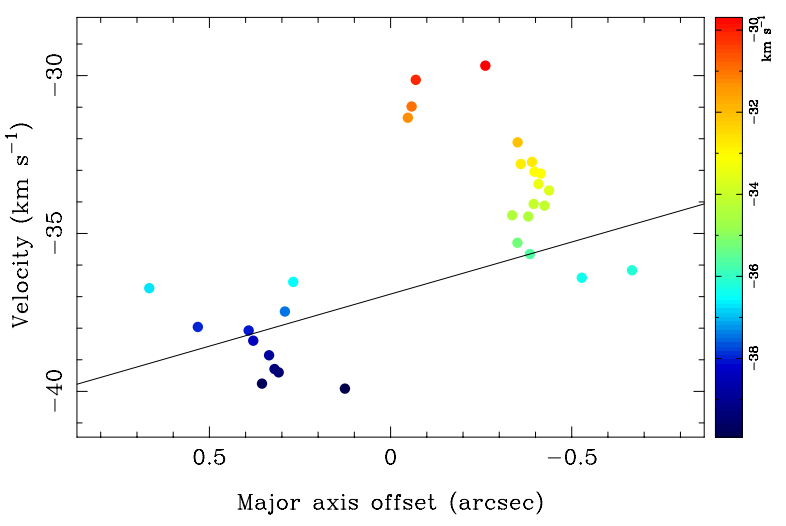

Fig. 3. Velocity-major axis plot, showing the distance along the major axis (the best fit to the disk) against velocity. The spots are extended in velocity perpendicular to the major axis, and the fit as a linear distribution due to emission from a ring around a central gravitational source which gives $11 M_{\odot}($ shown $)$ - is very poor.

some further emission in the extreme West around $-36.5 \mathrm{~km} \mathrm{~s}^{-1}$. The Eastern cluster appears at about $-36.5 \mathrm{~km} \mathrm{~s}^{-1}$ and runs in an easterly direction until around $-38 \mathrm{~km} \mathrm{~s}^{-1}$, after which the components appear to the South of the starting point.

Figure 3 plots the velocities of the features as a function of their distance along the major axis (shown as the line in Fig. 2) from the centre of the emission along this line, the velocities are along the y-axis. Emission from a thin ring in a disk would produce a linear distribution. The detailed mapping of spot position against velocity is difficult to reconcile with that expected from an edge-on Keplerian rotating disk model, as there is not a simple two sided structure let alone a monotonic increase in velocity along the main axis. But other models are as difficult to construct. If the emission delineates an entire disk, gravitationally bound by a massive central object, at a distance of $3 \mathrm{kpc}$, the implied enclosed mass is $11 M_{\odot}$. However, in this source the spots are extended across the major axis, as discussed in Dodson et al. (2004), and inconsistent with the disk-model. Similar structures are seen in the observations of water maser by Torrelles et al. (2001), on both the small and the large scale. Water masers unquestionably form in shocked out-flows. These facts combine to argue strongly against the disk-based models for the majority of the emission.

\section{Measuring the polarisation of $6.7 \mathrm{GHz}$ methanol maser spots}

From the polarised images we present fluxes and polarisation angles for all the spots, defined as regions of contiguous emission in space and velocity, in tabular form. Figures are also presented, showing the arrangement of the emission and the magnetic fields that this represents. The Northern region, being very weak, has no identifible polarised flux. The Western has rapidly varying linear polarisation angles $(\chi)$ which tend to follow the ridge of emission. Interestingly the direction undergoes a swap across the brightest point, changing by $90^{\circ}$ going North to South. Across the North-Eastern region, at the phase centre, $\chi$ is similar. In the most Eastern region $\chi$ is again variable. In the South-Eastern region $\chi$ the flux emitting region is continuous and $\chi$ is constant across this.

To relate $\chi$ to magnetic field direction is not simple. The dominant maser emission will either be parallel or perpendicular to the magnetic field lines, depending on the angle between the magnetic field and line of sight. If it is greater (less) than $\sim 55^{\circ}$ the vectors are perpendicular (parallel) to the magnetic field (Goldreich et al. 1973). Also when the masers are highly saturated there are further complications, but as the polarisation vectors are similar from all components, weak and strong, one can deduce that the emission is never sufficiently saturated to change the emitting regime. Furthermore, the source is extended on the sky, being over $1^{\prime \prime}$ across. It is unlikely, therefore, that the field is pointing towards us. The one region where this may not be correct is around the brightest spot in the Western cluster, where the polarisation vectors rapidly change by $90^{\circ}$ across it, yet the polarisation is zero at the centre. Very similar behaviour is also seen in the strongest feature reported on the $\mathrm{H}_{2} \mathrm{O}$ masers discussed in Vlemmings \& Diamond (2006). For our source better data is needed, in particular the magnetic field magnitudes, to solve for the full field behaviour using Stokes $V$. (As Methanol and $\mathrm{OH}$ masers are often found co-located (as in $\mathrm{W} 3(\mathrm{OH})$ Vlemmings et al. 2006) we could expect the field strengths of $\sim-4$ to $-6 \mathrm{mG}$ as found in Caswell (2003) and Caswell (2004) for $\mathrm{OH}$ masers at 6-GHz and $1720-\mathrm{MHz}$ respectivily.) Therefore we have assumed that we are in the regime where the polarisation vectors are perpendicular with the fields, and in all figures the polarisation vectors have been rotated by $90^{\circ}$, to show the magnetic field directions. Models for the 2D galactic magnetic field have recently been derived (Brown et al. 2007), so a line of sight rotation measure $(\mathrm{RM})(-7 \pm 3)$ can be deduced and used to solve for the magnetic fields. This low value for the RM is because the maser sits near a reversal in the galactic magnetic field, and all contributions cancel. The nearby pulsars J1625-4048 and J1703-4851 have RMs of -7 \pm 15 and $-4 \pm 24$. The model RM implies an angle change of +0.8 degrees at $6.7 \mathrm{GHz}$. This is less than our estimated errors (which are less than $5^{\circ}$ for all but the edges of the images), and so it has not been included.

Figure 4 shows the total flux in contours, with the vectors representing the magnetic fields, without the small correction for RM, scaled by the linear polarised flux intensity. The regions of interest are expanded. Figure 5 shows the South Eastern component from Fig. 4 but with the total flux as an image, the linear polarised in contours, and the vectors representing the magnetic field overlaid scaled with the fractional polarisation.

The major axis of the cluster lies at $-61^{\circ}$ (all angles are measured from north through east), and the majority of the vectors follow the ridge of emission, except for the reversal in the Western cluster, and the points to the south of the phase centre. The magnetic fields are expected to lie along the flow of material if the material is in a shock. For a disk the magnetic fields would be expected to thread through the disk and be at right angles to the major axis, which is clearly not the case across the majority of the emission. However, we note with interest that the region with the most continuous surface brightness, the Southern bar of the Eastern cluster, from a single narrow spectral feature spanning -38 to $-39.5 \mathrm{~km} \mathrm{~s}^{-1}$, has magnetic fields which lie as predicted by the disk model, that is perpendicular to the emission. See Fig. 5a in which Stokes I, overlaid with linear polarisation flux contours, and the vectors (rotated to represent the magnetic field) of the fractional polarisation and direction is plotted. The velocity-major axis plot (Fig. 5b) shows a linear relationship, but implies only a extremely small contained mass of $0.03 M_{\odot}$.

In Table 1 the data is summed (in $I, Q$ and $U$ ) over each "spot" in the full data cube, after masking for greater than $2 \mathrm{Jy} /$ beam in Stokes I, and the Stokes I, the linear (in janskys) and fractional (in percent) polarised flux measurement is 
R. Dodson: First VLBI observations of methanol maser polarisation, in G339.88-1.26

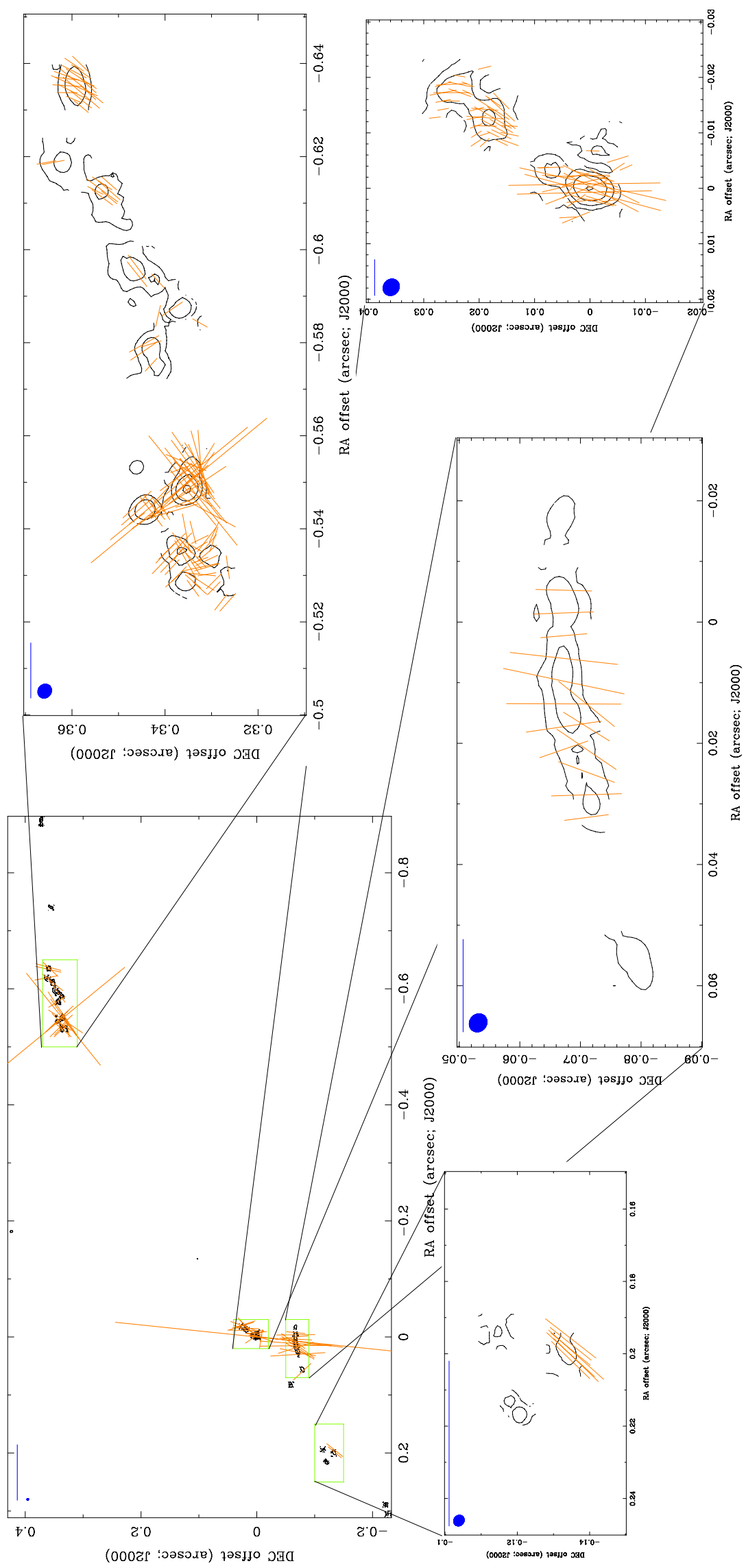

Fig. 4. Total flux (contours at 2, 8, 32 and $128 \mathrm{Jy} /$ beam) overlaid with the polarisation intensity and direction (scaled vectors). The beam size shown in top left, along with bar representing $10 \mathrm{Jy}$ of linearly polarised flux. The polarisation vectors are rotated to represent the magnetic field. The four regions with significant polarised emission are expanded. 
Table 1. Physical parameters for the emission in G339.88-1.26. Each region of emission, masked below $2 \mathrm{Jy} / \mathrm{beam}$ of total flux, is averaged over the channels and the integrated flux (Stokes $I$, and linear) was found. These are given in janskys (i.e. mean power in frequency for the region), and in percent. Where the flux found was less than $0.5 \mathrm{Jy}$ ( $4 \sigma$ for the linear polarised flux) no value is given. The size of the region (from a Gaussian fitted to the summed data) is given in mas and degrees. The maximum of this region is given in mas and $\mathrm{km} \mathrm{s}^{-1}$. The range of velocities over which the average flux was taken is given in $\mathrm{km} \mathrm{s}^{-1}$.

\begin{tabular}{rrrr|rrr|rrrr}
\hline \hline & \multicolumn{1}{c}{ Integrated flux } & \multicolumn{4}{c}{ Region size } & \multicolumn{3}{c}{ Position and velocity } \\
$I$ & linear & fraction & $\chi$ & Major & Minor & PA & $\Delta \alpha$ & $\Delta \delta$ & Peak & Range \\
Jy & Jy & $\%$ & $\circ$ & mas & mas & $\circ$ & mas & mas & $\mathrm{km} \mathrm{s}^{-1}$ & $\mathrm{~km} \mathrm{~s}^{-1}$ \\
3.3 & - & - & - & 4.0 & 2.0 & 15 & -182 & 424 & -30.1 & $-29.9--30.8$ \\
\hline 43.3 & - & - & - & 1.6 & 0.9 & 8 & -544 & 344 & -32.4 & $-31.9--32.9$ \\
22.2 & 2.2 & 10.1 & 57 & 13.7 & 5.6 & -66 & -612 & 353 & -33.3 & $-32.8--33.6$ \\
54.2 & 3.7 & 6.8 & 62 & 6.6 & 2.3 & -82 & -635 & 359 & -33.6 & $-33.3--34.2$ \\
43.5 & 1.5 & 3.5 & 75 & 7.9 & 4.5 & -60 & -619 & 362 & -34.0 & $-33.6--34.5$ \\
44.6 & 1.7 & 3.8 & -63 & 7.8 & 2.4 & -76 & -577 & 342 & -34.5 & $-34.2--34.9$ \\
135.7 & 2.0 & 1.5 & -32 & 12.6 & 8.5 & -46 & -534 & 335 & -34.9 & $-34.5--35.6$ \\
104.6 & - & - & - & 52.0 & 9.2 & -23 & -558 & 392 & -34.9 & $-34.9--35.6$ \\
244.7 & 1.2 & 0.5 & -71 & 2.0 & 1.1 & 87 & -549 & 335 & -35.4 & $-34.7--35.7$ \\
36.2 & 0.9 & 2.5 & -44 & 2.2 & 1.5 & -48 & -588 & 337 & -35.7 & $-35.4--35.9$ \\
59.0 & 1.8 & 3.0 & -81 & 26.3 & 1.5 & -88 & -887 & 372 & -36.3 & $-35.9--36.4$ \\
20.2 & 0.6 & 3.1 & 27 & 3.4 & 1.5 & -28 & -740 & 355 & -36.4 & $-36.1--36.8$ \\
\hline 148.0 & 6.9 & 4.7 & 80 & 14.8 & 4.0 & -43 & -14 & 20 & -36.6 & $-36.1--37.1$ \\
197.9 & 12.5 & 6.3 & 86 & 3.4 & 0.6 & -18 & 0 & 0 & -37.5 & $-37.0--38.0$ \\
\hline 39.5 & 1.8 & 4.6 & 29 & 10.2 & 5.3 & -61 & 306 & -227 & -36.8 & $-36.4--37.0$ \\
71.2 & 1.9 & 2.6 & 59 & 8.6 & 4.4 & -84 & 199 & -133 & -38.0 & $-37.7--38.4$ \\
\hline 78.2 & 1.5 & 1.9 & -46 & 7.9 & 3.7 & -61 & 56 & -79 & -38.4 & $-38.2--38.7$ \\
375.7 & 21.4 & 5.7 & 83 & 23.9 & 3.1 & -78 & 14 & -68 & -38.7 & $-38.4--39.3$ \\
55.8 & 4.0 & 7.2 & -76 & 8.4 & 3.2 & -76 & -3 & -67 & -39.1 & $-38.9--39.6$ \\
\hline
\end{tabular}
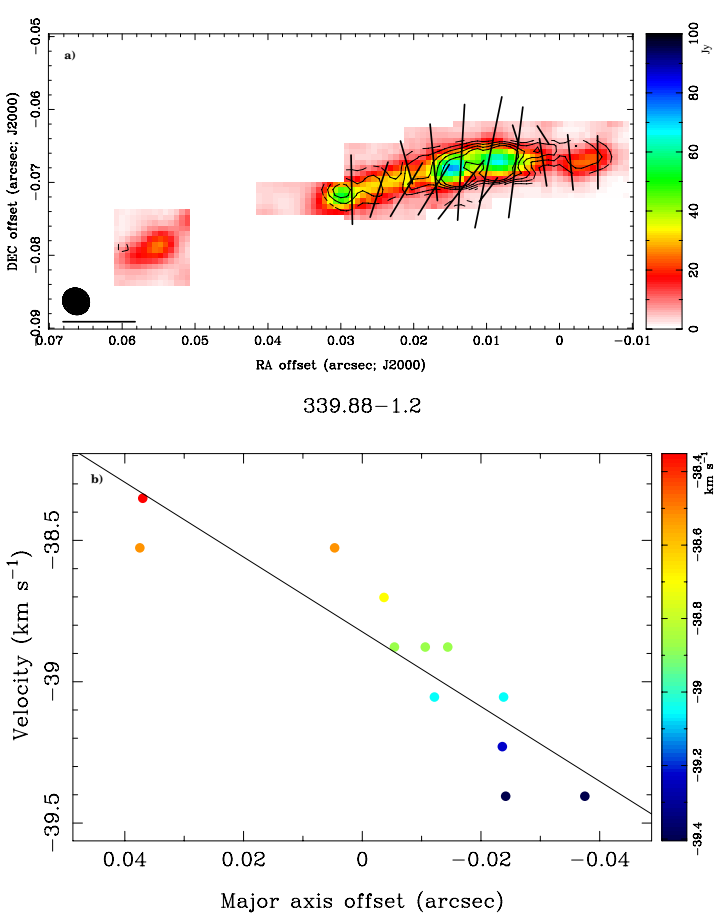

Fig. 5. a) Image of the SE feature with magnetic fields lying perpendicular to it. The image shows the total flux image, along with a colour bar, in Jy/beam. The linear polarised flux contours $(4,6,8,10$ and $12 \mathrm{Jy} / \mathrm{beam})$, and the polarisation vectors, scaled to the fractional polarised flux, rotated to represent the magnetic field direction. The beam size and a bar representing $10 \%$ fractional polarised flux is shown in the bottom left. b) The Velocity-Major Axis plot shows that this feature is linear in velocity space, and therefore could be represented by a ring (or partial ring) of emission around a central gravitational source.

derived. Furthermore a Guassian was fitted to the summed channels and the found extent (major and minor axes in mas and the position angle in degrees) is reported. This does not (except is the few cases where the emission is from a single component) represent the spot sizes, but the region over which emission is to be found. Finally the centre of the region and the velocity range (in mas for $\Delta \alpha$ and $\Delta \delta$, and $\mathrm{km} \mathrm{s}^{-1}$ for the velocity) is given.

\section{Discussion}

The debate for and against methanol masers forming in disks still rages a decade after it began. Higher resolution observations, IR and VLBI, are slowly throwing light onto the exact conditions of these sources. Certainly shock based models seem to be the only ones which can reproduce more than the most localised or gross features of methanol masers in massive star-forming regions. Even the ring of masers discovered by Bartkiewicz et al. (2005) do not fit neatly with the expectations of maser in a disk, despite the fact one of the major arguments against the disk model was the lack of top view masers. These masers also show a similar extension perpendicular to the major axis, in this case the ring around the central source. Nevertheless, the analysis of Pestalozzi et al. (2004), of one component of the maser emission in NGC 7538, indicates that probably disks do host some masers. Possibly a similar region is found in G339.88-1.26, in which the magnetic field lies parallel to the emission. However, in both cases, the majority of the source cannot be fitted in this fashion.

The extent of the SE bar (Fig. 5), given the distance of $3 \mathrm{kpc}$ based on the systematic velocity, is $200 \mathrm{AU}$ in size and only $1.5 \mathrm{~km} \mathrm{~s}^{-1}$ in velocity. This leads to a contained mass of $0.03 M_{\odot}$. This disk size is similar to what has been found in other star-forming regions, and if the region is made up of submassive stellar bodies, which will combine to form the eventual massive star, such an object is possibly feasible. However such an object could only survive where it was not disturbed by external turbulence, which is unlikely in a massive star-forming region. De Buizer et al. (2002) places the methanol masers on 
the edge of MIR source 1B, and suggest that they are formed by material flowing from that and interacting with $1 \mathrm{C}$. They suggest that an undetected (in MIR) foreground object is responsible for the radio and visible detections and estimate this to be of spectral type B2.5. One could assume, therefore, that this emission is from the front edge of a disk around such a star, as in Pestalozzi et al. (2004). The similarity of this object to the disk feature in NGC 7538 is striking, but as there are no good $12 \mathrm{GHz}$ images to compare with, the complete analysis performed by Pestalozzi et al. (2004) is not immediately possible.

The review of Vlemmings (2007) draws attention to the conclusions which can be drawn from the Stokes-V analysis of VLBI images. No attempt has been made to derive Stokes-V from the data, as we must firstly improve the polarisation purity. These are the first results in linear polarisation from the LBA, the polarisation corrections found are high, and it would be more sensible to use these results as a stepping stone to such interesting analysis.

\section{Conclusions}

The first polarisation results from the LBA, and the first VLBI polarisation images of methanol masers, are presented. The polarisation calibration of the LBA is undergoing development. This experiment, we believe, provides a first successful demonstration of the process. However, further work, on multiple targets, is required to provide complete confidence in the system. These observations will demonstrate the successful application of the calibration to a range of sources in the sky. Once this is complete the code will be incorporated in the AIPS distribution.

The target of our observation shows a disordered magnetic field, which in most regions lies along the ridge of emission. This is in accordance with shock generated features. However there is one small region in the complex which conforms to the expectations of a disk. The Position and Velocity-Major Axis plots (Fig. 5) show linearity in space and velocity and the magnetic field lies parallel to the feature. However it cannot represent a complete disk, as the deduced enclosed mass is extremely small $\left(0.03 M_{\odot}\right)$. Furthermore the narrow waist of NGC 7538 and evidence of an out-flow at right angles to the disk is missing in this case. Further observations, with improved polarisation calibration, and also at $12.2 \mathrm{GHz}$, are required to investigate this.

Acknowledgements. I wish to thank Dr Brown for calculating the RM for the direction and distance of this source, and Dr Ellingsen for making the ATCA polarisation data available. The referee, Dr Vlemmings, made comments which greatly improved the paper. This research has made use of NASA's Astrophysics Data System Abstract Service and the SIMBAD database, operated at CDS. The Long Baseline Array is part of the Australia Telescope which is funded by the Commonwealth of Australia for operation as a National Facility, managed by CSIRO and the University of Tasmania. I acknowledge support by a Marie Curie International Incoming Fellowship within the EU FP6 under contract number MIF1-CT-2005-021873. The Raman Research Institute provided hosting and support during the completion of this paper.

\section{References}

Aaron, S. 1997, EVN Memo, 78

Bartkiewicz, A., Szymczak, M., \& van Langevelde, H. J. 2005, A\&A, 442, L61 Batrla, W., Matthews, H. E., Menten, K. M., \& Walmsley, C. M. 1987, Nature, 326,49

Brown, J. C., Haverkorn, M., Gaensler, B. M., et al. 2007, ApJ, 663, 258

Caswell, J. L. 2003, MNRAS, 341, 551
Caswell, J. L. 2004, MNRAS, 349, 99

Caswell, J. L., Vaile, R. A., Ellingsen, S. P., Whiteoak, J. B., \& Norris, R. P. 1995, MNRAS, 272, 96

De Buizer, J. M., Walsh, A. J., Piña, R. K., Phillips, C. J., \& Telesco, C. M. 2002 , ApJ, 564, 327

Diamond, P. J. 1989, in Synthesis Imaging in Radio Astronomy, ed. R. A. Perley, F. R. Schwab, \& A. H. Bridle, ASP Conf. Ser., 6, 379

Dodson, R. 2007, On the solution of the polarisation gain terms for VLBI data collected with antennae having Nasmyth or E-W mounts, Informe Técnico OAN 2007-16, Observatorio Astronómico Nacional http: //www1. oan.es/ informes/archivos/IT-OAN-2007-16.pdf

Dodson, R., \& Rioja, M. 2008, On the astrometric calibration of mm-VLBI using dual frequency observations, Informe Técnico, OAN-2008-3, http: //www1. oan.es/informes/archivos/IT-OAN-2008-3.pdf

Dodson, R., Ojha, R., \& Ellingsen, S. P. 2004, MNRAS, 351, 779

Dos Santos, P. M., \& Lepine, J. R. D. 1979, Nature, 278, 34

Elitzur, M. 1996, ApJ, 457, 415

Ellingsen, S. P. 2002, in Cosmic Masers: From Proto-Stars to Black Holes, ed. V. Migenes, \& M. J. Reid, IAU Symp., 206, 151

Ellingsen, S. P., Norris, R. P., \& McCulloch, P. M. 1996, MNRAS, 279, 101

Forster, J. R., \& Caswell, J. L. 1989, A\&A, 213, 339

Furuya, R. S., Kitamura, Y., Wootten, H. A., Claussen, M. J., \& Kawabe, R. 2001, ApJ, 559, L143

Garay, G., \& Lizano, S. 1999, Publs. astr. Soc. Pacif., 111, 1049

Goedhart, S., Gaylard, M. J., \& van der Walt, D. J. 2004, MNRAS, 355, 553

Goldreich, P., Keeley, D. A., \& Kwan, J. Y. 1973, ApJ, 179, 111

Gray, M. D. 2003, MNRAS, 343, L33

Greisen, E. W. 1988, AIPS Memo, 61

Hollenbach, D., Johnstone, D., Lizano, S., \& Shu, F. 1994, ApJ, 428, 654

Koo, B.-C., Williams, D. R. D., Heiles, C., \& Backer, D. C. 1988, ApJ, 326, 931

Lin, D. N. C., \& Pringle, J. E. 1990, ApJ, 358, 515

MacLeod, G. C., Gaylard, M. J., \& Nicolson, G. D. 1992, MNRAS, 254, 1p

McKee, C. F., \& Tan, J. C. 2003, ApJ, 585, 850

Menten, K. M. 1991, ApJ, 380, L75

Menten, K. M., Reid, M. J., Moran, J. M., et al. 1988, ApJ, 333, L83

Menten, K. M., Reid, M. J., Pratap, P., Moran, J. M., \& Wilson, T. L. 1992, ApJ, 401, L39

Minier, V., Booth, R. S., \& Conway, J. E. 2000, A\&A, 362, 1093

Minier, V., Ellingsen, S. P., Norris, R. P., \& Booth, R. S. 2003, A\&A, 403, 1095

Moscadelli, L., Menten, K. M., Walmsley, C. M., \& Reid, M. J. 1999, ApJ, 519, 244

Mouschovias, T. C., \& Ciolek, G. E. 1999, in The Origins of Stars and Planetary Systems, ed. C. J. Lada, \& N. D. Kylafis, NATO Advanced Study Institute (Kluwer Academic Press)

Norris, R. P., Caswell, J. L., Gardner, F. F., \& Wellington, K. J. 1987, ApJ, 321, L159

Norris, R. P., Caswell, J. L., Wellington, K. J., McCutcheon, W. H., \& Reynolds, J. E. 1988, Nature, 335, 149

Norris, R. P., Whiteoak, J. B., Caswell, J. L., Wieringa, M. H., \& Gough, R. G. 1993, ApJ, 412, 222

Norris, R. P., Byleveld, S. E., Diamond, P. J., et al. 1998, ApJ, 508, 275

Pestalozzi, M. R., Elitzur, M., Conway, J. E., \& Booth, R. S. 2004, ApJ, 603, L113

Phillips, C. J., Norris, R. P., Ellingsen, S. P., \& McCulloch, P. M. 1998, MNRAS, 300,1131

Sault, R. J., Teuben, P. J., \& Wright, M. C. H. 1995, in Astronomical Data Analysis Software and Systems IV, ed. R. A. Shaw, H. E. Payne, \& J. J. E. Hayes, ASP Conf. Ser., 77, 433

Shepherd, M. C. 1997, in Astronomical Data Analysis Software and Systems VI, ed. G. Hunt, \& H. E. Payne, ASP Conf. Ser., 125, 6, 77

Stecklum, B., \& Kaufi, H. 1998, Circumstellar Disk around a Massive Star, ESO press release

Torrelles, J. M., Patel, N. A., Gómez, J. F., et al. 2001, ApJ, 560, 853

Vlemmings, W. H. T. 2007, ArXiv e-prints, 705

Vlemmings, W. H. T., \& Diamond, P. J. 2006, ApJ, 648, L59

Vlemmings, W. H. T., Harvey-Smith, L., \& Cohen, R. J. 2006, MNRAS, 371, L26

Walsh, A. J., Hyland, A. R., Robinson, G., \& Burton, M. G. 1997, MNRAS, 291, 261

Walsh, A. J., Burton, M. G., Hyland, A. R., \& Robinson, G. 1998, MNRAS, 301, 640

Watson, W. D. 1994, ApJ, 424, L37

Wilson, W. J., \& Barrett, A. H. 1972, A\&A, 17, 385 